\title{
Panorama da produção científica sobre Cuidados Intermediários e Hospitais Comunitários: uma revisão integrativa
}

\author{
Overview of scientific production on Intermediate Care and \\ Community Hospitals: an integrative review
}

Érika Fernandes Tritany', Túlio Batista Franco ${ }^{2}$, Paulo Eduardo Xavier de Mendonça'

DOI: $10.1590 / 0103-1104202112918$

RESUMO Mudanças epidemiológicas decorrentes do envelhecimento populacional motivaram transformações nos sistemas de saúde mundiais. Desde 1990, emergem discussões sobre Cuidados Intermediários (CI), visando diminuir hospitalizações, melhorar a coordenação do cuidado e estimular políticas públicas de cuidados próximos ao território. Este trabalho objetivou apresentar e analisar a literatura científica sobre CI, enfatizando serviços de Hospitais Comunitários. Realizou-se uma revisão integrativa, combinando os descritores "Intermediate Care", "Community Hospitals", "Intermediate Care Facilities", "Long-Term Care", "Hospitals Chronic Disease" e "Rehabilitation Services", nos idiomas português, inglês e espanhol, nas bases Portal Regional da Biblioteca Virtual em Saúde, PubMed e Biblioteca Digital Brasileira de Teses e Dissertações. Após processo de busca e elegibilidade, foram incluídos 30 documentos para síntese qualitativa, provenientes de: Reino Unido, Noruega, Espanha, Dinamarca, Austrália e Itália. Resultados apontam para a eficácia, a efetividade e a eficiência dos CI, com boas avaliações por parte de profissionais e usuários. Os CI, como estratégia de fortalecimento da Atenção Primária à Saúde, redução de internações, favorecimento da independência funcional dos pacientes e seu retorno à comunidade, apresentam-se como uma inovação em saúde e aposta promissora. Entretanto, persistem lacunas na literatura que ensejam mais estudos sobre o tema, com vistas a subsidiar a tomada de decisão.

PALAVRAS-CHAVE Envelhecimento. Instituições para cuidados intermediários. Hospitais Comunitários. Reabilitação. Serviços de reabilitação.

1 Universidade Federal do Rio de Janeiro (UFRJ) - Rio de Janeiro (RJ), Brasil. erika.tritany@gmail.com

2 Universidade Federal Fluminense (UFF) - Niterói (RJ), Brasil.

\begin{abstract}
Epidemiological changes resulting from populational aging have led to changes in global health systems. Since 1990, discussions on Intermediate Care (IC) have emerged, with a view to reducing hospitalizations, improving care coordination, and stimulating public care policies close to the territory. This work aims to present and analyze the scientific literature on CI, emphasizing services from Community Hospitals. An integrative review was carried out, combining the descriptors "Intermediate Care", "Community Hospitals", "Intermediate Care Facilities", "LongTerm Care", "Hospitals Chronic Disease", and "Rehabilitation Services", in Portuguese, English and Spanish, in the Regional Portal of Virtual Health Library, PubMed and Digital Library of Thesis and Dissertations bases. After the search and eligibility process, 30 documents were included for qualitative synthesis, coming from: The United Kingdom, Norway, Spain, Denmark, Australia and Italy. Results point to the efficacy, effectiveness and efficiency of ICs, with good evaluations by professionals and users. ICs, as a strategy to strengthen Primary Health Care, reduce hospital admissions, favor patients' functional independence and return to the community, are presented as a health innovation and promising bet. However, there are gaps in the literature that lead to more studies on the topic, with a view to supporting decision making.
\end{abstract}

KEYWORDS Aging. Intermediate care facilities. Hospitals, community. Rehabilitation. Rehabilitation services. 


\section{Introdução}

O cenário atual, nos serviços de saúde, apresenta desafios conflitantes aos hospitais: ao passo que cresce a demanda por atendimento e internações hospitalares, dadas as transições demográficas e epidemiológicas em curso, com aumento da população idosa e maior prevalência das doenças crônicas, crescem também pressões por diminuição de leitos, com vistas a atingir metas de eficiência. Como solução, tem sido apontada a diminuição do tempo de permanência das internações, o que pode comprometer o cuidado e a oportunidade de reabilitação das pessoas hospitalizadas. Os idosos, nesse cenário, aparecem como grupo mais vulnerável ante a possibilidade de serem mandados para casa de forma precoce $\mathbf{1}$.

A menor permanência no hospital é condicionada à existência de uma rede social estável da pessoa atendida, capacidade familiar ou do ente público para prover os cuidados necessários e adequados na própria residência; e à presença de serviços comunitários e de instituições de longa permanência para receber pessoas com necessidades de cuidados continuados e desprovidos de amparo social ${ }^{2}$.

No que tange à organização dos serviços de saúde, é frequente que o cuidado aos idosos opere a partir de um 'círculo vicioso': como reflexo das necessidades de saúde complexas, admissões hospitalares crescem; há uma diminuição no tempo de estadia, e, assim, as oportunidades para reabilitação são reduzidas. Isso impacta no uso de serviços domiciliares e diminuição de recursos para serviços preventivos, o que, por sua vez, culmina em mais internações hospitalares, retroalimentando o círculo ${ }^{3}$.

Esse cenário fortalece a necessidade de repensar apostas organizativas dos sistemas de saúde com o propósito de maior resolutividade dos problemas e necessidades de saúde, assim como eficiência no uso dos recursos ${ }^{4,5}$. Nesse sentido, o termo Intermadiate Care - Cuidados Intermediários (CI) - foi desenvolvido, e é cada vez mais utilizado, para descrever uma série de serviços destinados a facilitar a transição entre hospital e domicílio, impactando em uma redução nas admissões hospitalares e na construção de novos arranjos organizativos que intentam maximizar resultados em termos de ganhos em saúde 6 .

Assim, alguns exemplos de serviços de CI incluem: hospitais dia geriátricos (geriatric day hospitals); esquemas de hospital em casa e reabilitação domiciliar (hospital-at-home schemes and home-based rehabilitation); reabilitação domiciliar de enfermagem (nursing home-based rehabilitation); Hospitais Comunitários (HC) (Community Hospitals); equipes de resposta rápida (rapid response teams); Assistência Comunitária e Equipes de Reabilitação (Community Assessment and Rehabilitation Teams - Cart); Unidades Lideradas por Enfermeiros (nurse-led units) e serviços sociais como Centro de Reabilitação Dia (Day Centre Rehabilitation) e Centro Residencial de Reabilitação (Residential Care Rehabilitation) ${ }^{\mathbf{1}}$.

Os HC, por sua vez, apresentam-se como unidades de saúde mais próximas à comunidade; com estrutura física, equipe multiprofissional e processo de trabalho variáveis, mas com menor densidade tecnológica em comparação com hospitais gerais. Além disso, objetivam evitar internações desnecessárias ou fornecer apoio a pacientes após hospitalizações, com vistas à estabilização de seu quadro clínico e/ou melhorar sua independência para retorno ao domicílio. Assim, promovem maiores ganhos funcionais e independência pós-alta ${ }^{2,7,8}$. Entretanto, até onde foram nossas buscas, foi verificada escassez de estudos que abordem os HC como alternativa à hospitalização em Hospital Geral, sistematizem e apresentem diferentes experiências e evidências científicas'.

No Brasil, essa temática tem sido introduzida sob o conceito de Cuidados Prolongados, ganhando força com a Portaria $\mathrm{n}^{\circ} 2.809$, de 7 de dezembro de $2012^{9}$, mas ainda pouco explorada pela literatura científica.

Dessa forma, o objetivo deste trabalho foi caracterizar o estado atual da literatura científica sobre a temática dos $\mathrm{HC}$ e do modelo de 
CI a eles atrelado, apresentando e discutindo abordagens e modos de operação dos CI e dos $\mathrm{HC}$, bem como suas potencialidades e fragilidades apontadas pela literatura.

\section{Material e métodos}

Foi desenvolvida uma revisão integrativa da literatura, por consistir em uma metodologia de síntese do conhecimento científico. Tratase de uma abordagem mais ampla, quando comparada a outros formatos de revisão, pois permite a inclusão de estudos experimentais e não experimentais; dados da literatura teórica e empírica; e pode tratar de temas relacionados à definição de conceitos, revisão de teorias, evidências e análises metodológicas ${ }^{10}$.

\section{Critérios de elegibilidade}

Os critérios de elegibilidade orientaram o processo de seleção em todas as etapas. Por compreendermos que a denominação do serviço de CI pode sofrer variações entre países, foram considerados como HC instituições que apresentassem similaridades com os princípios, objetivos e modos de operação dos HC da Inglaterra, ainda que utilizassem outra nomenclatura, haja vista o pioneirismo desse país na normatização, na prestação e na disseminação de serviços de CI, o que influenciou sua implantação em outros países.

Os critérios de inclusão utilizados foram: 1) estudos relacionados à temática dos CI ou realizados em instituições de CI e $\mathrm{HC}$ e/ou que comparem instituições de CI com outros modelos de atenção à saúde, incluindo o $\mathrm{HC}$; 2) estudos disponíveis; 3) estudos publicados em qualquer ano, qualquer nacionalidade ou idioma e qualquer desenho de estudo.

Como critérios de exclusão, consideraram-se: 1) estudos realizados em instituições de CI em que, no entanto, o documento apenas cita a instituição, sem descrever suas características, não sendo possível identificar se atendem aos critérios de elegibilidade; 2) estudos exclusivamente relacionados ou realizados em domicílio, Instituições de Longa Permanência para Idosos (Ilpi), asilos de idosos, hospitais de cuidados agudos/hospitais gerais, Unidades Lideradas por Enfermeiros, Hospitais Dia/ Centro-Dia, Hospices, ou outra configuração de serviço intermediário sem o objetivo de estabelecer uma comparação entre diferentes modalidades assistenciais que inclua os $\mathrm{HC}$; 3) unidades de CI dentro de hospitais gerais ou relacionadas com alternativas à Terapia Intensiva; 4) documentos relativos a serviços voltados exclusivamente para Atenção Psiquiátrica, ainda que realizados em instituições de CI;

\section{Estratégia de busca bibliográfica}

Por sintetizarem as principais características do tema proposto, em sentido amplo, foram escolhidos os seguintes descritores DeCS/MeSH para compor a estratégia de busca: 1) "Cuidados Intermediários" "Intermediate Care"; 2) "Hospitais Comunitários" "Community Hospitals"; 3) "Instituições para Cuidados Intermediários" "Intermediate Care Facilities"; 4) "Assistência de Longa Duração" "Long-Term Care"; 5) "Hospitais de Doenças Crônicas" "Hospitals Chronic Disease"; 6) "Serviços de Reabilitação" "Rehabilitation Centers".

Apesar do termo "Cuidados Intermediários" "Intermediate Care" não se configurar como um Descritor em Ciências da Saúde pelo DeCS e MeSH, julgamos necessária sua utilização por ser a principal terminologia utilizada para referir esse modelo assistencial. Não foram incluídos termos correlatos aos CI, não classificados como descritores, o que poderia aumentar sobremaneira a sensibilidade dos resultados da busca e/ou focalizar os achados em experiências relacionadas com os países nos quais os termos são utilizados, em detrimento de outros países.

Foram criadas 14 chaves de busca - combinando os 6 termos citados com os operadores lógicos booleanos AND e OR - preenchidas na 
estratégia Busca Avançada nas bases de dados: Portal Regional da Biblioteca Virtual em Saúde (BVS), PubMed; e Biblioteca Digital Brasileira de Teses e Dissertações (BDTD), que reflete produções dos programas de pós-graduação no Brasil.

\section{Seleção dos documentos e extração dos dados}

O levantamento bibliográfico foi realizado em maio de 2019, sendo utilizado filtro 'documentos disponíveis' para todas as bases de dados, em consonância com o acordo internacional Budapest Open Access Initiative. Com esse acordo, foram apresentadas à comunidade científica recomendações para o desenvolvimento de políticas de acesso irrestrito e gratuito às pesquisas acadêmicas com o intuito de acelerar os esforços da pesquisa científica e atingir o maior número possível de leitores ${ }^{\mathbf{1 1}}$.

A estratégia de busca gerou muitos resultados, o que pode ter ocorrido pelo termo 'Intermediate Care' não ser classificado como descritor. Assim, inicialmente, foi realizada a leitura de todos os títulos, incluindo, para leitura do resumo, documentos que apresentassem termos correlatos ou que, por inferência, pudessem se referir ao tema. Foram excluídas as duplicidades, e procedeu-se à leitura dos resumos. Após, os documentos selecionados foram lidos na íntegra, sendo incluídos para extração dos dados e discussão dos resultados aqueles que atendessem aos critérios de elegibilidade.

Dos artigos incluídos, foram extraídos dados referentes a: autor; ano de publicação; país de origem; idioma de publicação; delineamento do estudo; formato do documento; objetivo do estudo e principais resultados, sintetizados na seção resultados e no quadro 1. A síntese qualitativa dos estudos incluídos foi apresentada na seção discussão, com base na sistematização e contraposição dos resultados e discussões emergentes dos estudos.

\section{Resultados}

Após seguir todas as etapas de seleção, foram incluídos 30 documentos. Os resultados do levantamento bibliográfico estão descritos no fluxograma da figura 1 . 
Figura 1. Fluxograma da informação com as diferentes fases da revisão

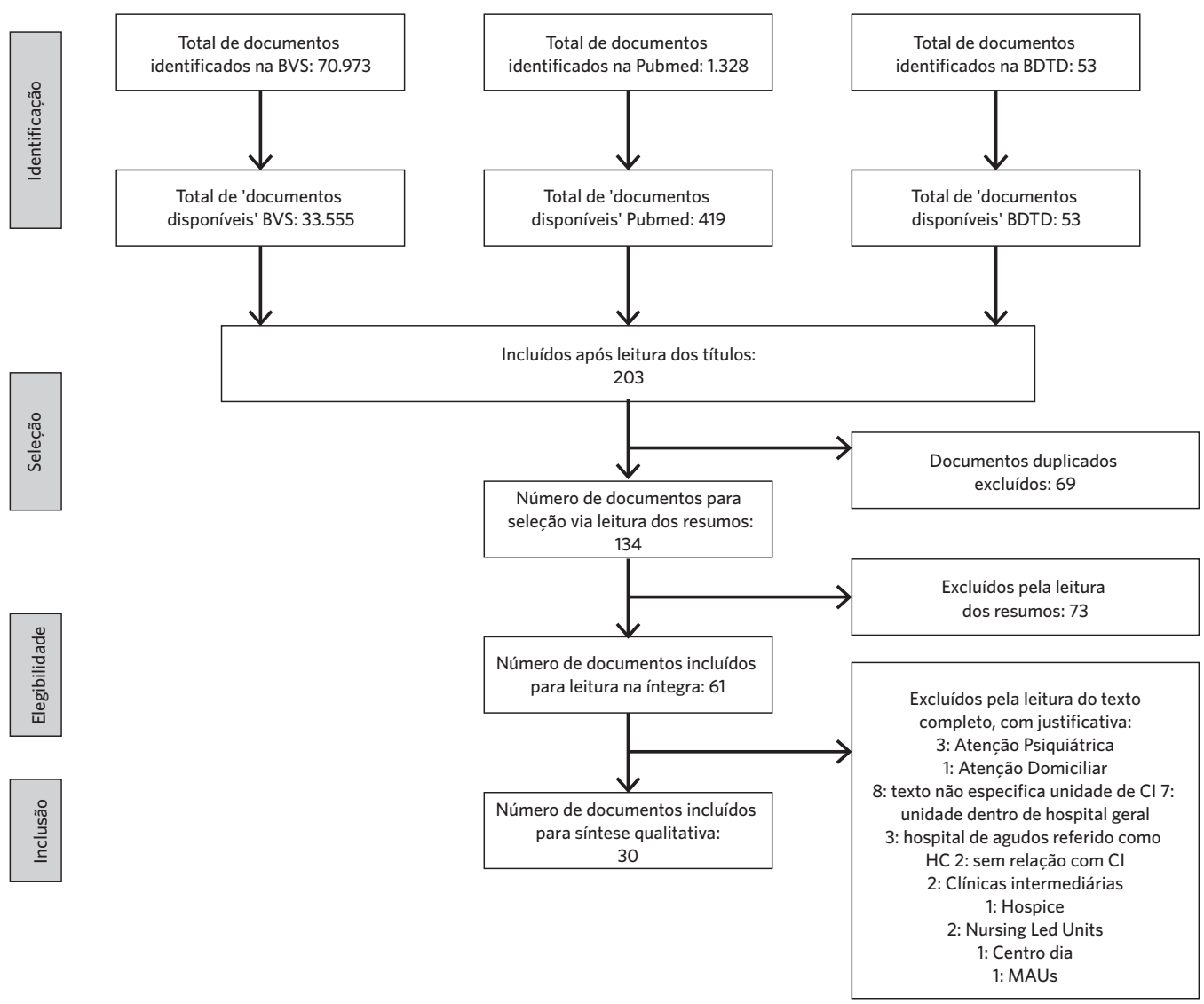

Fonte: Elaboração própria.

O quadro 1 sintetiza os 30 documentos incluídos. Os estudos foram majoritariamente provenientes do Reino Unido (17 estudos, $56,6 \%)$, sendo: 4 da Inglaterra $(13,3 \%)^{8,12-14} ; 1$ da Escócia (3,3\%) 1 ; do País de Gales (3,3\%) ${ }^{\mathbf{1 5}}$; 1 da Irlanda do Norte $(3,3 \%)^{16}$. Os demais não especificaram o país de origem 1,3,17-24. Além disso, 6 estudos noruegueses foram incluídos $(20 \%)^{2,7,25-28} ; 4$ da Espanha $(13,3 \%)^{29-32} ; 1$ da Dinamarca (3,3\%) ${ }^{33}$; 1 da Austrália $(3,3 \%)^{34}$ e 1 da Itália $(3,3 \%)^{35}$. O idioma predominante foi o inglês, com 27 documentos (90\%); havendo também 2 documentos em espanhol $(6,6 \%)^{30,32}$ e 1 em norueguês $(3,3 \%)^{27}$.
Em relação ao desenho de estudo, foram identificados 4 ensaios clínicos randomizados (13,3\%) e 2 não randomizados $(6,6 \%)$ (sendo 1 protocolo de pesquisa); 5 coortes (16,6\%); 6 estudos seccionais (20\%) (sendo 1 protocolo de pesquisa); 5 estudos qualitativos (16,6\%); 2 revisões narrativas (6,6\%); 2 estudos de caso (6,6\%) e 4 comentários (13,3\%), denotando representatividade de diferentes métodos de análise e discussão da temática. A maioria dos documentos estava publicada em formato de artigo original completo (22; 73,3\%), mas 8 tratava-se de relatos breves

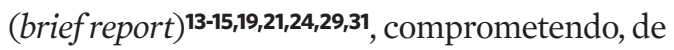
certa forma, a qualidade do relato das pesquisas. 
Quadro 1. Artigos selecionados para compor a revisão - extração dos dados

\begin{tabular}{llll}
\hline Autor/Ano & Desenho de Estudo & País de origem / idioma & Objetivo \\
\hline Higgs, 198512 & Estudo de caso & Inglaterra/ Inglês & Apresentar o Lambeth \\
& & Hospital, reformado, em \\
& & 1985, como uma unidade \\
& & comunitária de Cl.
\end{tabular}

Seamark et al., Estudo Seccional Reino Unido/ Inglês 200120

$\begin{array}{lll}\text { Grant; Dowell, } & \text { Estudo de Caso } & \text { Escócia/Inglês } \\ 2002^{6} & \text { Qualitativo } & \end{array}$

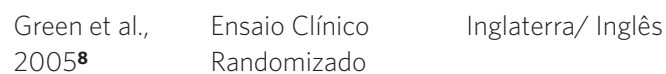

Vetter, $2005^{\mathbf{1 4}}$ Comentário

Garasen; Win- Ensaio Clínico dspol; John- Randomizado sen, $2007^{2}$
País de Gales, Reino Unido/ Inglês

Noruega/ Inglês .
Conhecer o perfil dos $\mathrm{HC}$ do Reino Unido. $\begin{array}{ll}\text { Garasen; Win- } & \text { Ensaio Clínico } \\ \text { dspol; John- } & \text { Randomizado } \\ \text { sen, 20087 } & \end{array}$

Garasen et al., Ensaio Clínico $2008^{26} \quad$ Randomizado

Kaambwa et Estudo Seccional Reino Unido/Inglês

\section{Noruega/ Inglês}

Principais Resultados

O centro possuía 20 leitos e 1 unidade dia para atendimento de pacientes em processo de terminalidade; cuidados paliativos; que necessitassem de cuidados agudos não especializados; com incapacidades e/ou pós-operatório.

Foram identificados $471 \mathrm{HC}$ no Reino Unido, totalizando 18.579 leitos, divididos em leitos gerais, de idosos, cirúrgico, maternidade, cuidados paliativos, cuidados pós-operatórios e de enfermagem. Foram identificados 7425 clínicos gerais em Cl, 20\% do total de General Practitioners (GP).

Identificar fatores que influenciam as decisões dos clínicos gerais (GP) sobre a admissão em $\mathrm{HC}$.

Comparar HC e hospital geral em relação à independência de idosos após a alta.

Analisar a política de Cl do National Health Service.

Comparar a eficácia dos HC com cuidados 'padrão' em um hospital geral, após 26 semanas de seguimento.

Noruega/ Norueguês

Comparar resultados pacientes atendidos em um HC com aqueles em tratamento tradicional em hospital geral.

Comparar custos de internação em um hospital geral com um centro intermediário configurado como $\mathrm{HC}$.

Avaliar custos e resultados associados a diferentes tipos de serviços de $\mathrm{Cl}$ e analisar as características dos pacientes que recebem esses serviços.
Todos os GP relataram benefícios aos pacientes com o uso do HC. Seis temas principais orientaram as decisões dos GP: capacidade do hospital; capacidade do médico; e preferência do paciente; preocupações profissionais; influências pessoais; e benefícios potenciais.

O grupo tratado no HC apresentou maior independência nos seis meses após a internação em comparação com o grupo do hospital geral.

É sugerida cautela na adoção dos $\mathrm{Cl}$ como política pública de saúde uma vez que sua segurança não está comprovada.

Comparado ao hospital geral, o $\mathrm{HC}$ reduziu significativamente o número de reinternações para a mesma doença $(19,4 \%$ no grupo intervenção e $35,7 \%$, controle; $p=0,03)$; aumentou o número de pacientes independentes de cuidados comunitários e domiciliares (25\% no grupo intervenção e 10\%, controle; $p=0,02)$, com fracas evidências de aumento no tempo de permanência e sem aumento da mortalidade.

O grupo intervenção foi observado por um período maior (335,7 dias HC e 292,8 dias no hospital geral). Não houve diferenças para uso de cuidados no nível primário, reinternações ou dias em leitos hospitalares.

Os custos no HC foram menores que os custos do hospital geral. Diferenças podem ser atribuídas ao maior número de reinternações pelo grupo do hospital geral e menores custos do tratamento no $\mathrm{HC}$.

47\% dos pacientes analisados foram admitidos inapropriadamente em serviços de Cl. Serviços de evitação da admissão foram associados a menores custos e maiores ganhos funcionais e de saúde. Os ganhos são maiores quando há maior a gravidade da doença, dependência dos pacientes e admissão apropriada. 
Quadro 1. (cont.)

\begin{tabular}{|c|c|c|c|c|}
\hline Autor/Ano & Desenho de Estudo & País de origem / idioma & Objetivo & Principais Resultados \\
\hline $\begin{array}{l}\text { Glasby; Mar- } \\
\text { tin; Regen, } \\
2008^{3}\end{array}$ & Estudo Qualitativo & Reino Unido/Inglês & $\begin{array}{l}\text { Analisar percepção dos } \\
\text { profissionais sobre a rela- } \\
\text { ção entre serviços hospita- } \\
\text { lares e } \mathrm{Cl} \text {. }\end{array}$ & $\begin{array}{l}\text { Destaca tensões entre } \mathrm{Cl} \text { e cuidados agudos. Ques- } \\
\text { tões-chave incluem preocupações sobre o papel } \\
\text { e o envolvimento dos médicos de hospitais gerais } \\
\text { em } \mathrm{Cl} \text {; segurança, qualidade e adequação dos } \mathrm{Cl} \\
\text { para idosos; acesso e elegibilidade para os Cl; falta } \\
\text { de compreensão e sensibilização dos profissionais; } \\
\text { e o risco de compartilhar o cuidado entre níveis de } \\
\text { atenção diferentes. }\end{array}$ \\
\hline
\end{tabular}

Regen et al., Estudo Qualitativo Reino Unido/Inglês 200819

Yohannes;

Coorte

Baldwin; Con-

nolly, $2008^{22}$

Reino Unido/ Inglês
Analisar percepção dos profissionais sobre a implementação dos $\mathrm{Cl}$ na Inglaterra.

Young, 20091 Revisão Narrativa Reino Unido/ Inglês

Dixon et al., Estudo Seccional Inglaterra / Inglês $2010^{11}$

Inzitari et al., Coorte $2012^{29}$
Espanha/ Espanhol
Narrativa sobre o desenvolvimento dos $\mathrm{Cl}$ na Inglaterra, suas características gerais e evidências que apoiam sua eficácia.

Avaliar a relação entre o 'mix de habilidades', os resultados dos pacientes, tempo de permanência e custos dos serviços de $\mathrm{Cl}$ na Inglaterra.

Avaliar alta precoce de pacientes com doenças crônicas reativadas a partir de um hospital universitário e emergência para um hospital intermediário de convalescença.
Principais desafios: escassez de mão de obra e de financiamento, dificuldade de trabalho em conjunto entre agências de saúde e assistência social e falta de envolvimento por parte dos médicos. Benefícios aos usuários: centralidade do cuidado no paciente e promoção da independência, sendo trabalho em equipe multiprofissional, flexibilidade de papéis e ambiente "caseiro" e acolhedor os principais benefícios. Principais desafios: capacidade insuficiente, problemas de acesso e coordenação deficiente entre $\mathrm{Cl}$ e hospital.

173 pacientes, com idade média de 80 anos, sendo 60 homens. 130 (75\%) sofreram uma ou mais quedas no ano anterior. 65 pacientes (38\%) apresentavam sintomas depressivos; 29 (17\%), depressão clínica; 73 (43\%), sintomas de ansiedade, e 43 (25\%), ansiedade clínica. Dos que apresentavam depressão clínica, 14 (48\%) foram considerados levemente, e 15 (52\%), moderadamente deprimidos. A estada mais longa ocorreu pela severidade da depressão, inabilidade física, baixa cognição e pelo paciente viver sozinho.

Apesar dos $\mathrm{Cl}$, os hospitais gerais ainda vivem pressões, e houve aumento nas internações agudas. Evidências científicas apontam para o modelo de Hospital at Home como a melhor alternativa de serviços de $\mathrm{Cl}$. Os HC também se mostram uma alternativa viável.

O aumento do mix de habilidades, pelo aumento da variedade de profissionais da equipe, está associado a uma redução de 17\% dos custos dos serviços $(p=0,011)$. Fracas evidências $(p=0,09)$ de que $o$ aumento na proporção de pessoal de apoio promova melhor qualidade de vida.

Foram encaminhados para $\mathrm{Cl} 68$ pacientes (média de 82,6 anos; $48,5 \%$ homens), $69,1 \%$ provenientes do serviço de urgência. Diagnósticos principais: doenças cardíacas (23,5\%); respiratórias (51,5\%); e crônicas descompensadas. O tempo médio de permanência em $\mathrm{Cl}$ foi 11,4 dias. Após a alta, 82,4\% dos pacientes regressaram ao local de origem; 4,4\% foram a óbito; 2,9\% reingressaram a serviços de urgência ou hospitais e 10,3\% necessitaram de outros recursos sociossanitários. 
Quadro 1. (cont.)

\begin{tabular}{|c|c|c|c|}
\hline Autor/Ano & Desenho de Estudo & País de origem / idioma & Objetivo \\
\hline $\begin{array}{l}\text { Colprim et al., } \\
2013^{28}\end{array}$ & $\begin{array}{l}\text { Ensaio clínico não } \\
\text { randomizado }\end{array}$ & Espanha/Inglês & $\begin{array}{l}\text { Comparar idosos interna- } \\
\text { dos diretamente em uma } \\
\text { Unidade Geriátrica de } \mathrm{Cl} \\
\text { (ICGU), a partir de casa, } \\
\text { com pacientes da mesma } \\
\text { área geográfica que recebe- } \\
\text { ram alta do hospital geral } \\
\text { para a mesma ICGU. }\end{array}$ \\
\hline
\end{tabular}

Santaeugènia Carta ao editor - Espanha/ Inglês et al., $20133^{30} \quad$ Estudo Seccional
Apoiar os resultados de Colprim et al.28 com base em sua própria experiência em ICGU, e apresentar dados transversais desta unidade.

Analisar a qualidade percebida dos pacientes atendidos em um HC para auxiliar no desenvolvimento de alternativas para os hospitais gerais.

Apresenta resultados do Relatório da Auditoria Nacional de Cuidados Intermediários da Inglaterra, de 2013, e discute os desafios à prestação dos $\mathrm{Cl}$.

Comparar a eficácia das altas hospitalares de pacientes com mais de 60 anos em dois municípios: um com hospital de $\mathrm{Cl}$ e outro, sem.

\section{Principais Resultados}

65 pacientes (média de idade $\pm 85,6 \pm 7,2,66 \%$ mulheres) foram admitidos, em 2011, na ICGU, principalmente por insuficiência cardíaca (55,4\%), doença pulmonar obstrutiva crônica (20\%) e doença renal crônica (9,2\%): 32, diretamente de casa, e 33, após alta hospitalar aguda. Os grupos foram comparáveis para características clínicas, funcionais e sociais na admissão e desfechos de alta, incluindo mortalidade e transferências agudas. O tempo de permanência global foi maior para pacientes oriundos da internação hospitalar $(60,8$ $\pm 26,6$ vs $38,4 \pm 23$ dias, $p<0,001)$. Apontam internações diretas em ICGU como alternativa à hospitalização aguda.

201 pacientes foram internados na ICGU (dez/2009-jul/2012). Idade média 84,2 anos; Índice de Barthel 40; cognitive impairment em 56\%; $87 \%$ dos pacientes oriundos do departamento de emergência e 13\% diretamente da comunidade. As principais condições agudas de admissão foram infecção respiratória (57\%), insuficiência cardíaca (18\%), infecção urinária (10\%) e outros (15\%). Duração média da estadia na unidade foi 10.4 dias. Taxa de readmissão pelo mesmo motivo em 30 dias e de utilização de recursos de saúde foi, respectivamente, 5,5\% e 7,8\%. As ICGU apresentam-se como alternativa à hospitalização de pacientes idosos.

Pacientes do $\mathrm{HC}$ relataram maior satisfação que os atendidos no hospital geral. Os fatores relativos ao HC foram: ambiente silencioso e acolhedor; proximidade com a comunidade; continuidade na relação entre profissionais e usuários. Aspectos abrangentes ressaltados: abordagem interdisciplinar e holística; apropriação e proximidade com as práticas locais; cooperação com serviços especializados.

Problemas de capacidade estavam relacionados com variação de configuração, tamanho e desempenho entre os serviços; maior ênfase em serviços de alta apoiada do que de evitação da admissão; lacunas na formação de profissionais para habilidades de $\mathrm{Cl}$, saúde mental e demência.

328 pacientes internados no hospital geral de referência para os dois municípios foram acompanhados durante um ano. O município com hospital de $\mathrm{Cl}$ apresentou menor tempo de internação hospitalar e o mesmo risco de readmissões, mortalidade e necessidades de cuidados pós hospitalização e habilitação para AVD que o município sem um hospital de $\mathrm{Cl}$. 
Quadro 1. (cont.)

\begin{tabular}{|c|c|c|c|c|}
\hline Autor/Ano & Desenho de Estudo & País de origem / idioma & Objetivo & Principais Resultados \\
\hline $\begin{array}{l}\text { Dahl et al., } \\
2015^{24}\end{array}$ & $\begin{array}{l}\text { Coorte retrospec- } \\
\text { tiva }\end{array}$ & Noruega/ Inglês & $\begin{array}{l}\text { Comparar a utilização } \\
\text { de serviços de saúde por } \\
\text { pacientes com mais de } 60 \\
\text { anos em dois municípios: } \\
\text { um com hospital de Cle } \\
\text { outro, sem. }\end{array}$ & $\begin{array}{l}\text { Diminuição no tempo de internação hospitalar no } \\
\text { município com } \mathrm{Cl} \text {, sem diferenças nas readmissões } \\
\text { hospitalares durante o ano de acompanhamento. } \\
\text { No ano após a alta hospitalar, o município com } \mathrm{Cl} \\
\text { ofereceu mais horas de cuidados primários para } \\
\text { pacientes idosos que moravam em casa; o muni- } \\
\text { cípio comparativo apresentou maior permanência } \\
\text { prolongada em asilos. }\end{array}$ \\
\hline $\begin{array}{l}\text { Martinsen; } \\
\text { Norlyk; Lom- } \\
\text { borg, } 2015^{32}\end{array}$ & Estudo Qualitativo & Dinamarca/ Inglês & $\begin{array}{l}\text { Explorar a experiência de } \\
\text { idosos em uma unidade de } \\
\text { Cl após internação hos- } \\
\text { pitalar. }\end{array}$ & $\begin{array}{l}\text { A essência de estar na unidade de Cl foi concebida } \\
\text { pelos idosos como "momentos de alívio condicio- } \\
\text { nal" constituído das seguintes questões: "acessível, } \\
\text { abraçando o cuidado"; "uma corrida contra o tem- } \\
\text { po"; "refeições - convenções com modificações"; } \\
\text { "contato em termos desiguais"; "vida em outros } \\
\text { termos" e "ajuda informal, mas essencial". }\end{array}$ \\
\hline $\begin{array}{l}\text { Young et al., } \\
2015^{23}\end{array}$ & $\begin{array}{l}\text { Artigo de Opinião/ } \\
\text { Comentário }\end{array}$ & Reino Unido/Inglês & $\begin{array}{l}\text { Discutir avanços e desafios } \\
\text { dos } \mathrm{Cl} \text { na Inglaterra. }\end{array}$ & $\begin{array}{l}\text { A Auditoria Nacional de } \mathrm{Cl} \text {, de 2013, cobriu apro- } \\
\text { ximadamente metade dos serviços do NHS na } \\
\text { Inglaterra. Há uma variação local na provisão de } \\
\text { serviços de } \mathrm{Cl} \text {, e a capacidade de atendimento das } \\
\text { necessidades da população é estimada em cerca } \\
\text { de metade. Há baixa integração dos Cl com outros } \\
\text { serviços de saúde e assistência social. O relato dos } \\
\text { usuários é positivo, mas requer maior envolvimento } \\
\text { dos pacientes em decisões assistenciais. }\end{array}$ \\
\hline $\begin{array}{l}\text { Milte et al., } \\
2015^{33}\end{array}$ & Estudo Qualitativo & Austrália/ Inglês & $\begin{array}{l}\text { Descrever os encontros } \\
\text { entre idosos, cuidadores e } \\
\text { geriatras em Cl e explorar } \\
\text { características associadas à } \\
\text { comunicação do processo } \\
\text { de Tomada de Decisão } \\
\text { Compartilhada (TDC) }\end{array}$ & $\begin{array}{l}\text { Foram avaliadas } 59 \text { reuniões familiares envolvendo } \\
\text { geriatras, familiares e pacientes em um serviço de } \\
\mathrm{Cl} \text { após admissão hospitalar aguda. O tempo de } \\
\text { duração da reunião foi considerado importante } \\
\text { para TDC. Temas mais discutidos foram: problemas } \\
\text { médicos }(n=59) \text {, medicamentos ( } n=59) \text {, planeja- } \\
\text { mento avançado de cuidados }(n=55) \text { e destino da } \\
\text { alta }(n=53) \text {. }\end{array}$ \\
\hline $\begin{array}{l}\text { Millar et al., } \\
2016^{15}\end{array}$ & Estudo transversal & $\begin{array}{l}\text { Reino Unido - Irlanda do } \\
\text { Norte/ Inglês }\end{array}$ & $\begin{array}{l}\text { Compreender a conscienti- } \\
\text { zação e envolvimento dos } \\
\text { farmacêuticos comunitários } \\
\text { com os serviços de } \mathrm{Cl} \text {. }\end{array}$ & $\begin{array}{l}\text { Questionários postais foram respondidos por } 190 \\
\text { Farmacêuticos Comunitários, ( } 35,3 \% \text { de 539). } \\
\text { 47,4\% relataram entender o termo Cuidado Inter- } \\
\text { mediário. Três quartos não sabiam se estavam en- } \\
\text { volvidos com prestação de Cl. Apenas 9,5 e 0,5\% } \\
\text { concordaram fortemente que a comunicação do } \\
\text { hospital e Cl, respectivamente, foi suficientemente } \\
\text { detalhada. Poucos farmacêuticos (1,2\%) foram } \\
\text { comunicados sobre mudanças de prescrições em } \\
\text { hospitais ou Cl "todo o tempo". 81,6\% relataram } \\
\text { desejar maior envolvimento com Cl, sendo "carga } \\
\text { de trabalho atual" a maior barreira. }\end{array}$ \\
\hline $\begin{array}{l}\text { Gladman et } \\
\text { al., } 2016^{16}\end{array}$ & $\begin{array}{l}\text { Protocolo de Pes- } \\
\text { quisa }\end{array}$ & Reino Unido/ Inglês & $\begin{array}{l}\text { Medir custo-eficiência } \\
\text { da reabilitação em HC; } \\
\text { identificar características } \\
\text { dos HC que otimizam seu } \\
\text { desempenho e o impacto } \\
\text { social de sua otimização; } \\
\text { examinar a relação entre Cl } \\
\text { e atenção secundária. }\end{array}$ & $\begin{array}{l}\text { O estudo ainda não apresenta resultados. Não } \\
\text { foram citados resultados esperados. }\end{array}$ \\
\hline
\end{tabular}


Quadro 1. (cont.)

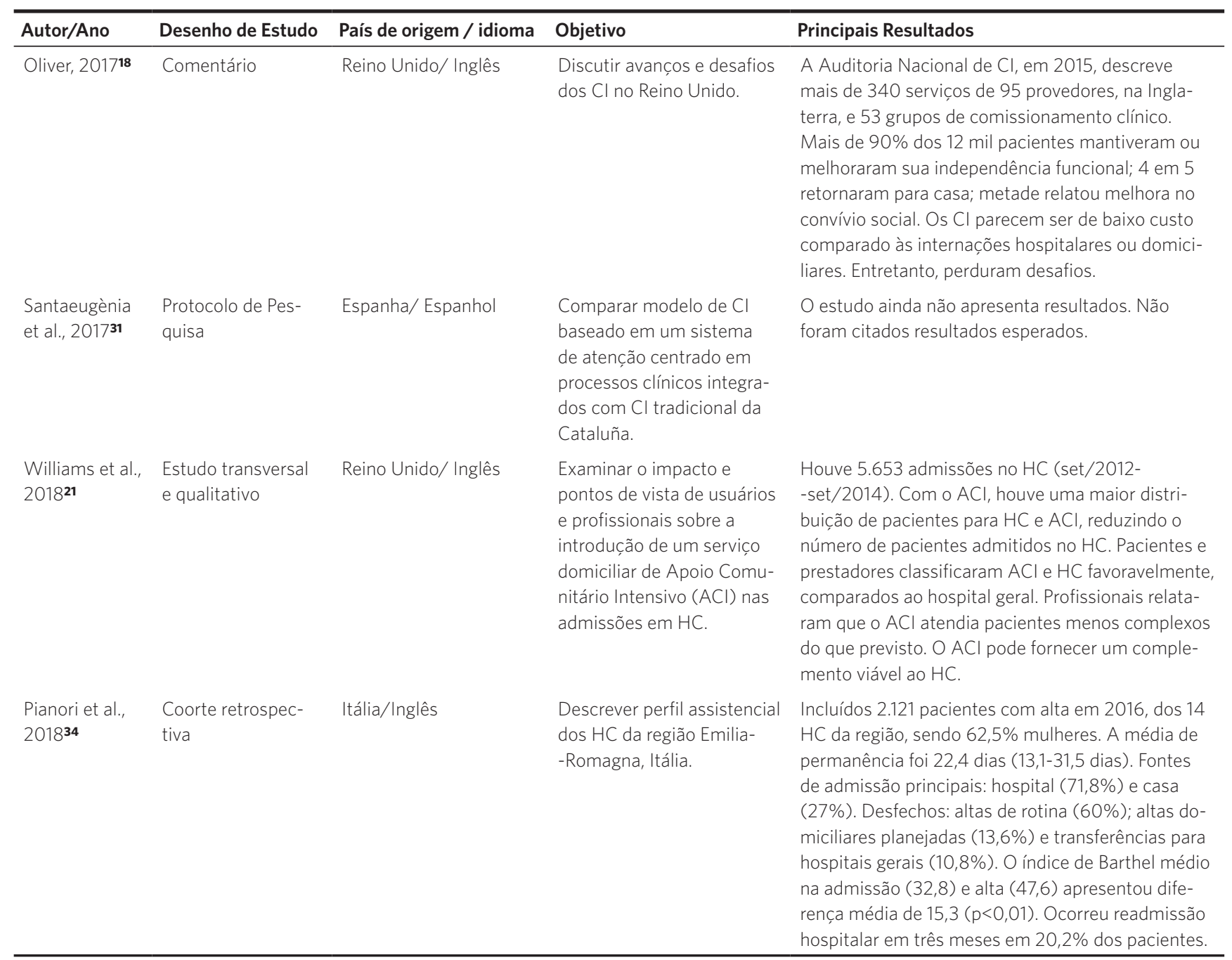

Fonte: Elaboração própria.

\section{Discussão}

\section{Os Cuidados Intermediários}

Os CI apresentam diferenças na disposição, oferta e características dos serviços de acordo com particularidades regionais e do país em que se desenvolveram.

No Reino Unido, combater o atraso na alta hospitalar surgiu, desde a década de 1990, como uma prioridade. Para tanto, foi introduzida uma série de medidas que ampliaram o financiamento para saúde e assistência social e incluíram novas legislações, com a finalidade de liberar leitos ocupados por pessoas que aguardam a prestação de serviços sociais. São enfatizados, também, o foco na redução das internações hospitalares de emergência e o apoio a pessoas com condições crônicas e de cuidados de longo prazo 3 .

$\mathrm{Na}$ Inglaterra, o conceito de CI foi proposto pela primeira vez no National Beds Inquiry, publicado em 2000, tornando-se uma política de cuidados do National Health Service (NHS) nesse ano e implementado como parte 
do National Service Framework (NSF) para pessoas idosas em 2001. Assim, foi introduzida uma série de serviços de CI em toda a Inglaterra, e financiadas mais de 6.700 vagas de $\mathrm{CI}^{1}$, número que foi crescendo ao longo dos anos. Entretanto, estima-se ainda que sua capacidade instalada corresponda a apenas metade das necessidades apresentadas pela população $0^{14,19,24}$.

Para definição dos CI, foram propostos diversos conceitos. Com vistas a subsidiar o seu monitoramento, realizado a partir de Auditorias Nacionais na Inglaterra, os serviços de CI foram descritos como aqueles destinados a pessoas para as quais a admissão hospitalar aguda mostra-se inadequada ou desnecessária ou para promover a continuidade assistencial após uma internação, caracterizando serviços de 'evitação da admissão' e 'alta apoiada' respectivamente. Os serviços, limitados temporalmente, normalmente não superior a seis semanas, estariam baseados em uma avaliação abrangente do paciente e trabalho em equipe multiprofissional, com enfoque individual, compreendendo terapia ativa, tratamento e reabilitação a fim de maximizar a independência, permitir o retorno do usuário ao domicílio e evitar ao máximo a admissão dos pacientes em asilos e lares de idosos. O planejamento e a implementação desses serviços ficaram a cargo da atenção primária e dos serviços sociais'

Apesar de a literatura indicar o desenvolvimento histórico dos CI a partir da década de 1990, encontramos em nossas buscas um estudo que descreve uma unidade hospitalar da Inglaterra, fechada em 1976 e reinaugurada em 1985 como uma unidade comunitária de CI, a qual apresentava características físicas e assistenciais próprias aos $\mathrm{HC}^{\mathbf{1 3}}$. Isso sugere a existência prévia dessas estruturas nos serviços de saúde, normatizadas e popularizadas, entretanto, posteriormente; abrindo espaço para que os CI fossem incorporados como política pública de diversos países.

Na Noruega, o sistema de saúde e assistência social é principalmente público, e a prestação de serviços de saúde ocorre a partir de um modelo descentralizado. Os hospitais são estatais e organizados por empresas de saúde administradas por quatro autoridades regionais de saúde. Os municípios são responsáveis pelos serviços de atenção primária, incluindo atendimentos de emergência, assistência domiciliar, casas de enfermagem/lares de idosos, HC, parteiras, fisioterapeutas, terapeutas ocupacionais; e pela gestão dos General Practitioners (GP) - Clínicos Gerais ${ }^{\mathbf{2}, 26,28}$. O governo da Noruega, por sua vez, possui e administra hospitais gerais distritais, hospitais universitários e serviços de ambulância por meio das autoridades regionais de saúde nas cinco regiões do País ${ }^{2}$.

A Reforma Nacional de Coordenação, implementada na Noruega em 2012, objetivou fortalecer serviços municipais de atenção primária e reabilitação. Assim, fomentou a criação de serviços de saúde que visassem substituir as internações ou sucedê-las. Entretanto, as diretrizes não trouxeram especificações sobre padrões de qualidade, suas implicações, formas de avaliação e critérios de elegibilidade de pacientes: as recomendações incluem pacientes estáveis com diagnóstico esclarecido ou aqueles que necessitam de observação ou investigação básica, mas com quadro clínico estável${ }^{28}$.

$\mathrm{Na}$ Espanha, as Unidades Geriátricas de Atendimento Intermediário (ICGU) objetivam encurtar hospitalizações agudas e evitar aquelas desnecessárias, baseadas em avaliação geriátrica abrangente e reabilitação ${ }^{29}$. As ICGU são utilizadas para atender pacientes com reativações de doenças crônicas que não podem ser tratadas em ambiente doméstico por necessitarem de monitoramento mais frequente ou por dificuldades sociais no manejo da situação em casa. Podem ser admitidos a partir do Hospital ou por mecanismos de admissão direta, sob coordenação da atenção primária29,31.

Está previsto, no Plano Diretor Sociossanitário do Departamento de Saúde da Catalunha, referência rápida, para unidades intermediárias, de pacientes oriundos de urgências e hospitais gerais, selecionados por especialistas em geriatria (médico, enfermeira 
e assistente social). Os critérios de elegibilidade considerados são clínicos (pelo diagnóstico principal dado pelo médico) ou sociais (pacientes em situação estável, com clara orientação diagnóstica e sem necessidade de exames complexos). Preconiza-se uma média de permanência na atenção intermediária não superior a 16 dias; retorno ao domicílio habitual para mais que $70 \%$ dos pacientes; mortalidade menor que $10 \%$ dos pacientes; reingresso a urgências ou hospitais gerais menor que $5 \%$; e referência a outro recurso sociossanitário para menos de $15 \%$ dos pacientes ${ }^{30}$.

$\mathrm{Na}$ Dinamarca, país onde a taxa de envelhecimento populacional segue acelerada, hospitais e serviços comunitários possuem financiamento público. Programas de reabilitação baseados na comunidade são fornecidos por meio de um seguro público de saúde. Com o objetivo de proteger os idosos e evitar altas precoces, o governo dinamarquês investiu em CI, como parte importante de sua política de saúde, com objetivo de melhorar serviços de saúde pública para idosos e reduzir gastos em saúde ${ }^{33}$.

Os CI oferecem fisioterapia, terapia ocupacional e cuidados planejados individualmente, preenchendo a lacuna entre hospital e casa, objetivando promover recuperação mais rápida, prevenir internações hospitalares agudas desnecessárias, apoiar a alta hospitalar e propiciar maior independência às pessoas em tratamento ${ }^{33}$.

Na Itália, a primeira menção acerca de serviços de CI ocorreu nas diretrizes do Plano Nacional de Saúde de 2006 a 2008. Desde então, as regiões do País, independentes administrativamente, vêm optando por diversas modalidades estratégicas de implementação desses serviços ${ }^{35}$.

No Brasil - embora não tenhamos encontrado resultados de experiências brasileiras -, a Portaria $\mathrm{n}^{\circ} 2.809$, de 7 de dezembro de 2012, estabelece a organização dos Cuidados Prolongados como retaguarda à Rede de Atenção às Urgências e Emergências (RUE) e demais Redes de Atenção à Saúde, a partir de Unidades de Internação em Cuidados Prolongados dentro de Hospitais Gerais ou em Hospitais Especializados em Cuidados
Prolongados. Busca-se fortalecer a integralidade pela articulação com equipes de saúde atuantes no território, em especial, a Atenção Primária à Saúde (APS) ${ }^{9}$.

\section{Processo de trabalho, gestão e organização da atenção nos Hospitais Comunitários}

Os HC são hospitais locais, unidades ou centros cujo objetivo é fornecer cuidados de saúde à comunidade com vistas a atender suas necessidades. Apresentam-se como uma extensão dos cuidados primários. Há uma grande ênfase nos cuidados de reabilitação (ofertada por 97\% dos HC do Reino Unido), mas os HC também podem oferecer cuidados paliativos, serviços de promoção da saúde, diagnóstico, emergência e serviços terapêuticos de diversas naturezas ${ }^{17,21}$.

Os HC são responsáveis pelo atendimento de pacientes que necessitam de: 1) intervenções de saúde que poderiam ser providas em casa, mas existem fatores estruturais e domiciliares que impedem sua elegibilidade para atendimento domiciliar; 2) necessidade de vigilância contínua de enfermagem; 3) premência de reabilitação ou estabilização do quadro clínico antes do retorno ao domicílio. $\mathrm{O}$ acesso pode ocorrer a partir de casa ou de instalações residenciais, por referência do médico de família; a partir das altas hospitalares ou diretamente do setor de emergência. Os cuidados serão prestados 24 horas por pessoal de enfermagem, clínicos gerais, pediatras de livre escolha e médicos de cuidados contínuos, além da presença da equipe de reabilitação, variável em cada localidade. A localização física do HC pode ser adequadamente alocada para enfermarias e/ ou pela conversão de instalações residenciais ${ }^{35,36}$.

No Reino Unido, os HC apresentam configurações bastante diversas: no que tange à provisão de leitos por 100 mil habitantes, há uma variação de menos de 10 a 70; sobre liderança clínica, $50 \%$ das unidades são lideradas por enfermeiros, e 50\%, por consultores; o tempo médio de internação varia de 11 a 58 dias; o custo por admissão pode atingir valores entre $£ 3.700$ e $£ 17.500$; e custo por dia de $£ 140-450$, sendo apontado potencial 
para melhoria na eficiência, por menor custo ou tempo de permanência, impactando em mais atendimentos e/ou economia para os serviços. As razões por trás dessas variações e os caminhos necessários para melhoria do desempenho dos serviços são, entretanto, especulativos, pois nenhum estudo foi conduzido nesse sentido ${ }^{17}$.

O HC localizado na cidade de Bradford, Inglaterra ${ }^{8}$, apresentava 18 leitos. A equipe multiprofissional era composta por enfermeiros, assistentes de enfermagem, fisioterapeutas, terapeutas ocupacionais, auxiliares de terapia (therapy helper), nutricionista (uma visita semanal), fonoaudiólogo (uma visita semanal), médico hospitalar (três visitas semanais), consultor geriatra (duas visitas semanais), e visitas dos GP fora do horário. Os pacientes, ao ingressar na instituição, eram avaliados pela equipe e recebiam um plano de cuidados individualizado com o propósito de maximizar sua recuperação e independência. Eram realizadas avaliações domiciliares antes da alta, que era coordenada com o serviço social local.

Na Noruega, muitos HC são comparáveis com os da Inglaterra e com hospitais de clínica geral da Holanda ${ }^{2}$. Outros países, como Itália $^{35}$; Escócia6; Espanha ${ }^{30}$; Dinamarca ${ }^{33} \mathrm{e}$ Austrália ${ }^{34}$, também apresentam essa modalidade assistencial - além de outros países não identificados em nossas buscas na literatura, como Portugal e Brasil, por exemplo.

O HC Lar de Søbstad, na Noruega ${ }^{2}$, foi baseado em cuidados individualizados, incluindo avaliação e tratamento das doenças de cada paciente com enfoque no processo de reabilitação para retorno ao domicílio. Para admissão no HC pelo médico da instituição, era realizada a avaliação dos pacientes e dos registros de saúde mais antigos disponíveis (do hospital geral e assistência domiciliar), além de comunicação com o paciente e sua família para compreender suas necessidades e o nível de atenção mais adequado, levando-se em consideração as preferências de ambos.

Lappegard e Hjortdahl28 estudaram a instituição Hallingdal Sjukestugu (HSS), instituição comparável aos HC da Grã-Bretanha, localizado em uma região rural ao sul da Noruega com 20 mil habitantes em 6 municípios. O HSS era administrado pelo hospital geral mais próximo, a $170 \mathrm{~km}$ de distância. Possuía uma unidade de internação com 14 leitos, ambulatórios somáticos e psiquiátricos, 1 centro de tratamento diurno com diálise e cuidados paliativos, 1 unidade para raio-x digital e 1 base para helicópteros e ambulâncias. Era administrado por dois GP, que contavam com apoio de especialistas do hospital geral via telefone, telemedicina ou pessoalmente. Recebia cerca de 600 internações anuais. Em 2012, os diagnósticos mais frequentes acolhidos foram pneumonia, exacerbações de doença crônica, trauma sem necessidade cirúrgica, dor e agravamento dos sintomas do câncer; com média de permanência de 4,8 dias.

Embora nem sempre a denominação dos serviços e da política seja realizada da mesma forma entre os países, as características de sua provisão, organização do processo de trabalho e concepções se assemelham, bem como a responsabilidade municipal ou distrital na condução desses serviços e provimento de aconselhamento especializado necessário. Foi observada uma maior frequência de desenvolvimento de $\mathrm{HC}$ menores, com poucos leitos (de 12 a 20) e menor quantitativo profissional2,8,26,28,35. Entretanto, foram também identificadas experiências de serviços maiores, com mais de 270 leitos de convalescença geriátrica, como a experiência da Catalunha ${ }^{29-31}$.

\section{Resultados promissores para Cuidados Intermediários e Hospitais Comunitários}

A partir dos estudos analisados, depreende-se que os $\mathrm{HC}$ podem representar um menor uso dos leitos hospitalares, entretanto, o tempo de utilização total dos leitos (hospital geral e HC) pode ser maior, o que pode ser justificável caso seja evidenciada maior qualidade do cuidado e resultados clínicos $^{8}$. São apoiados em evidências de alta qualidade quanto a sua eficácia ${ }^{2,7,8} \mathrm{e}$ custo-efetividade, por apresentarem melhores 
desfechos com uso semelhante de recursos ${ }^{24}$.

Também são notáveis resultados que apontam para benefícios inerentes aos $\mathrm{HC}$ e aos CI, como: ganhos funcionais importantes para pacientes durante a estada no $\mathrm{HC}^{35}$; menores taxas de readmissão hospitalar e maior número de pacientes não dependentes de atendimento comunitário/serviços domiciliares em relação àqueles tratados no hospital geral. O diálogo contínuo com os profissionais da APS foi considerado elemento central na redução de reinternações precoces, pela otimização do acompanhamento pós-alta ${ }^{\mathbf{2} 26}$.

Em relação aos custos da assistência intermediária, quando comparada à alternativa hospitalar padrão, foi identificada, por alguns autores, economia nos gastos totais em saúde²7; ao passo que outros citaram melhores resultados de saúde com uso semelhante de recursos ${ }^{24}$.

Embora esquemas de alta apoiada (internação hospitalar seguida de admissão em serviço intermediário) sejam mais frequentes ${ }^{14,24,35}$, a atuação dos HC como serviços de evitação da admissão (admissão direta em CI) foi avaliada como uma alternativa viável e segura às internações hospitalares ${ }^{29}$, e associada a menores custos e maiores ganhos funcionais e de saúde, quando comparada à alta apoiada ${ }^{18}$.

No entanto, vale destacar que todos os estudos incluídos nesta revisão que se propuseram analisar e comparar os custos da assistência intermediária com o atendimento hospitalar e com outras modalidades de serviços intermediários relataram importantes limitações metodológicas que evidenciam a necessidade de mais pesquisas que averiguem se, de fato, há uma economia inerente a essa prestação assistencial ou se os custos dos serviços se assemelham ${ }^{\mathbf{1 2 , 1 8 , 2 7}}$.

Ganham ênfase boas experiências relatadas pelos usuários ${ }^{33} \mathrm{e}$ boa avaliação por parte dos profissionais ${ }^{6,20}$. A satisfação do usuário pelo atendimento intermediário é ressaltada, tanto pelo sentimento de gratidão, pelo cuidado amigável e individual provido ${ }^{33}$ quanto pelo ambiente acolhedor que permitiu relação mais estreita entre profissionais, pacientes e família, dada a proximidade com a comunidade; diferentemente do hospital, descrito como um ambiente agitado e apressado, além da fragilidade do vínculo e distanciamento entre equipe e usuários, sem estímulo à reabilitação. Outrossim, a maioria dos pacientes relatou abordagem mais holística no $\mathrm{HC}$, que prestava atendimento de modo abrangente, com vistas à integralidade e atento às especificidades de cada pessoa ${ }^{28}$. O trabalho em equipe multiprofissional também foi avaliado de forma bastante positiva pelos usuários - que se sentiam seguros e estimulados a aderir ao treinamento de reabilitação - e pelos profissionais, que reconheciam os benefícios aos pacientes e se viam motivados e desafiados pelo compartilhamento de funções e responsabilidades 20,33.

É enfatizado o ambiente dos serviços intermediários como propício à interação social na medida em que algumas unidades apresentavam espaços coletivos para realização das refeições e outras atividades de lazer, caso fosse preferência dos pacientes. Isso trazia à tona dificuldades, como ser confrontado com a própria incapacidade e com a do outro; mas favorecia momentos de conversação entre pacientes e cuidadores e compartilhamento de experiências, que podem configurar-se como estímulos à sociabilidade, podendo promover diminuição da ansiedade sobre o retorno à casa e ao ambiente social, bem como auxílio na superação dos desafios inerentes ao processo de reabilitação ${ }^{33}$.

O desenvolvimento de um plano individual de cuidados demonstrou-se um mecanismo para favorecer maior recuperação e independência dos pacientes visando menor institucionalização das pessoas 1,3,8,20,24,33. A comunicação dos profissionais com a pessoa atendida e sua família, para tomada de decisão de admissão, buscando compreender suas percepções e preferências acerca do nível de atenção mais adequado às suas necessida$\mathrm{des}^{2}$, foi apontada pelos autores como potente estratégia para o favorecimento da tomada de decisão compartilhada ${ }^{34}$. Essas questões enfatizam benefícios inerentes a essa modalidade assistencial. 


\section{Desafios aos Cuidados Intermediários e Hospitais Comunitários}

Apesar dos benefícios apontados pela introdução dos CI e HC, algumas questões precisam ser levadas em consideração uma vez que limitações e incertezas ainda pairam sobre esses serviços, e são enfatizadas com importância na literatura.

A despeito da premissa de economia de gastos, a busca por reduções no tempo de internação hospitalar levanta preocupações acerca de que altas precoces possam aumentar readmissões e taxas de mortalidade. Ensaios clínicos evidenciam resultados diversos sobre a utilização dos HC: ao passo que estudos apontam para a redução da permanência com redução da mortalidade , diminuição das reinternações e aumento da independência de cuidados domiciliares e comunitários pós-alta ${ }^{2}$; bem como efeitos positivos na recuperação das Atividades de Vida Diária (AVD) seis meses após alta do $\mathrm{HC}^{\mathbf{8}}$; há também achados que não encontram diferenças significativas para desfechos pós-alta ${ }^{26}$.

Além disso, perduram incertezas sobre a relação custo-efetividade da prestação de serviços intermediários, bem como dúvidas sobre de onde provém a economia relatada pelos estudos que a defendem ${ }^{24}$, o que enseja o fortalecimento de processos de avaliação econômica em saúde.

Essas questões apontam para dificuldades na consolidação de uma base empírica que apoie a adoção dos CI como política pública, haja vista um número limitado de estudos publicados que apresentem fortes evidências científicas de sua eficácia e eficiência, com base na realização de Ensaios Clínicos Randomizados (ECR), Revisões Sistemáticas (RS), metanálises e estudos de avaliação econômica. Além disso, limitações no delineamento e na condução dos estudos comprometem a acurácia e a reprodutibilidade dos achados, e deixam dúvidas quanto à segurança e à eficácia da adoção desse modelo de atenção à saúde ${ }^{15}$, o que fortalece a importância da realização de
RS sobre o tema, com avaliação do risco de viés dos estudos selecionados (atualmente, pelo instrumento Risk of Bias - ROB-II).

Associado a isso, a existência de uma diversidade de definições e configurações de serviços de CI dificulta processos de avaliação institucionalizados pelo governo local e comparações inter-regionais e internacionais, além do estabelecimento de diretrizes clínicas baseadas em evidências, visando ao aprimoramento da qualidade dos serviços e dos resultados obtidos ${ }^{28}$.

A falta de uma normatização clara sobre critérios para admissão em CI e responsabilidades dos profissionais e níveis de atenção envolvidos ${ }^{\mathbf{2 8}}$ acarreta um processo de admissão baseado em critérios subjetivos, assim como propicia envolvimento variável dos profissionais de saúde com os serviços de CI, principalmente por parte dos Clínicos Gerais responsáveis pelo encaminhamento a esses serviços ${ }^{6}$. Além disso, pode favorecer admissões inadequadas em CI, o que impacta em menores ganhos funcionais pela pessoa em processo de reabilitação ${ }^{\mathbf{1 8}}$.

Os autores relataram ser frequente, nos serviços, uma compreensão equivocada dos profissionais sobre os CI, que acabam por utilizar suas instalações para 'liberar leitos hospitalares' em detrimento da escolha do ambiente de cuidados capaz de maximizar a recuperação e o retorno ao domicílio em segurança, com base em avaliação abrangente, necessidades e preferências de cada $\mathbf{u m}^{\mathbf{2 0}}$.

Essa questão também é reflexo de baixas integração e coordenação entre os diferentes serviços, o que compromete o potencial dos CI para aliviar pressões dos serviços assistenciais e sociais ${ }^{20}$ e favorece um cenário de comunicação deficiente entre profissionais, sobretudo entre diferentes níveis de atenção ${ }^{16}$, e atrasos no acesso aos serviços de $\mathrm{CI}^{19}$.

São enfatizadas, também, dificuldades relacionadas com o recrutamento e retenção de profissionais de saúde e pessoal de apoio às equipes de CI. Financiamento insuficiente e problemas para atrair profissionais para os cargos foram 
apontados como as principais causas ${ }^{20}$. Como impedimentos profissionais e barreiras ao recrutamento, foram destacados: potencial isolamento profissional dentro de pequenas equipes de trabalho; baixa conscientização acerca dos CI; baixos salários e longas horas de isolamento ou com baixa sociabilidade ${ }^{20}$.

Em relação à experiência dos pacientes, foram também detectadas deficiências no envolvimento do paciente com planejamento de metas, tomada de decisões assistenciais e envolvimento dos cuidadores e familiares; além de ter sido destacado sentimento de insegurança pela alta não ter sido discutida ou ter ocorrido sem aviso ${ }^{\mathbf{2 4}}$.

Por fim, faz-se notar que, na própria concepção do modelo de atenção intermediária, há um distanciamento de questões pertinentes à saúde mental. Além de haver pouco envolvimento dos profissionais de CI no tratamento de pessoas com complicações relacionadas à saúde mental (com poucos serviços que aceitam pacientes com complicações psiquiátricas) e baixa proporção de capacitações aos profissionais de saúde para atendimento a pacientes com demência ou outros problemas de saúde mental ${ }^{14,24}$, é intrigante que não tenha sido citada, em nenhum dos estudos analisados, a presença dos profissionais de psicologia na equipe de reabilitação de CI, o que pode representar uma invisibilidade dessas questões no cuidado em saúde e na negligência aos potenciais impactos que isso possa causar no tempo de permanência institucional e no processo de reabilitação ${ }^{23}$.

A reabilitação deve ser entendida é um processo complexo, adaptado à situação de saúde de cada pessoa, e buscar resultados multidimensionais, como a funcionalidade global e os parâmetros de qualidade de vida ${ }^{36}$. Assim, a reabilitação deve ser percebida de maneira ampla desde seu aspecto conceitual, bem como em suas considerações técnicas e instrumentais, realizada de maneira transdisciplinar, por meio de intervenções planejadas sobre todas as dimensões do cuidado (física, emocional, social e espiritual), com seu foco na tríade do cuidado - paciente, família e equipe multiprofissional ${ }^{36}$.

\section{Limitações desta revisão}

O número reduzido de bases de dados pesquisadas e a não inclusão de documentos contidos nas referências bibliográficas dos artigos analisados podem representar uma limitação do potencial que esta revisão poderia alcançar. Outrossim, a não utilização de termos correlatos aos CI, em nossa estratégia de busca, pode ter ocasionado a não obtenção de estudos sobre experiências brasileiras e/ou de outras nacionalidades.

\section{Considerações finais}

A partir desta revisão integrativa, de modo pioneiro, obteve-se um panorama geral da produção científica sobre o tema dos HC e do modelo de CI próprio a eles, possibilitando identificar características gerais desse nível de atenção à saúde; modo como é abordado na literatura e nos sistemas de saúde; desenhos de estudo mais frequentes; seus resultados positivos, desafios e lacunas ainda inexploradas pela literatura, o que abre espaço para a realização de novas pesquisas.

\section{Implicações para a prática}

Os CI, como estratégia de fortalecimento da APS, melhoria da independência funcional dos pacientes e favorecimento de seu retorno à comunidade, apresentam-se como uma inovação nos cuidados em saúde e como uma aposta promissora aos governos.

Pensar na reorganização dos serviços de saúde de modo a atender às necessidades de saúde, a diminuir internações inadequadas, a promover ganhos funcionais e maior qualidade de vida é uma tarefa urgente para governantes de diferentes nacionalidades. A busca por sistemas de saúde universais, equânimes, pautados em boas práticas clínicas, integralidade, no cuidado 
centrado na pessoa e na promoção dos projetos de felicidade deve ser um imperativo frente as ondas neoliberais que invadem o pensamento estatal e promovem o desmonte institucional de valores orientados ao bem comum.

Nesse sentido, aprender com a experiência já vivida por outrem possibilita alçar voos maiores na busca por aprimoramento de nossas iniciativas, bem como respeitar e endossar valores exprimidos na Reforma Sanitária. É importante que as características positivas dos CI, aqui expressas, sejam exaltadas e contrapostas a outros modelos organizativos com intenção de adequar a oferta, a organização e a gestão dos serviços de saúde vigentes, levando em consideração ressalvas e lacunas existentes no conhecimento científico sobre o tema.

Para atingir seu potencial, os CI devem estar integrados a um continuum assistencial em que se relacionem todos os níveis de atenção à saúde e assistência social, de modo regionalizado e hierarquizado, em prol da integralidade. Nesse sentido, o fortalecimento de mecanismos de coordenação e de integração da Rede de Atenção à Saúde é um passo importante e implica o estabelecimento de desenhos mais claros de protocolos e diretrizes assistenciais; investimento na formação e qualificação profissional; e compartilhamento de responsabilidades assistenciais. A implementação de políticas públicas de fortalecimento da APS e de serviços comunitários e territorializados apresenta-se, assim, como uma aposta promissora.

Merece também destaque a importância dos cuidados paliativos como abordagem de cuidado transversal aos serviços de saúde, fortalecendo uma concepção multidimensional da saúde orientada para assistência às dimensões física, emocional, social e espiritual da dor e do sofrimento ${ }^{37,38}$. O reforço dessa abordagem nos serviços de CI e demais níveis assistenciais da rede pode favorecer modificações no paradigma do cuidado em saúde, bem como possibilitar a prestação de serviços de saúde orientados aos usuários e suas necessidades de saúde, familiares e sua rede de apoio social, como também à equipe profissional envolvida no cuidado ${ }^{39}$.

\section{Implicações para pesquisas futuras}

Novas pesquisas devem ser fomentadas a fim de preencher importantes lacunas do conhecimento.

É importante conhecer a fundo princípios e características de provisão dos CI, ensejando a criação de um Modelo Teórico dos CI, que subsidie processos de implantação, monitoramento e avaliação desses serviços.

Estimulamos a realização de estudos sobre experiências brasileiras com CI e investigações sobre a implantação dos cuidados prolongados no Brasil.

Outro enfoque de pesquisa orienta-se à aplicação de métodos sólidos e confiáveis de avaliação de custos no campo da saúde nos serviços de CI, com o intuito de investigar sua (suposta) eficiência.

Outrossim, não identificamos estudos que explorem variações na configuração dos $\mathrm{HC}$ nos diferentes países, seus resultados; e caminhos para seu aprimoramento e/ou comparações entre países.

\section{Agradecimento}

A Roberto José Gervasio Unger, pelo compartilhamento de saberes fundamentais para delineamento metodológico deste estudo.

\section{Colaboradores}

Tritany EF (0000-0002-7099-4800)* contribuiu para concepção e planejamento do projeto; análise e interpretação dos dados; redação; revisão crítica do conteúdo do manuscrito e aprovação da versão final. Franco TB (0000-0001-7372-5262)* contribuiu para revisão crítica do conteúdo e aprovação da versão final. Mendonça PEX (0000-00026925-3693)* contribuiu para planejamento do projeto, revisão crítica do conteúdo e aprovação da versão final.
*Orcid (Open Researcher and Contributor ID). 


\section{Referências}

1. Young J. The development of intermediate care services in England. Arch Gerontol Geriatr. 2009; (49):S21$-\mathrm{S} 25$.

2. Garåsen H, Windspoll R, Johnsen R. Intermediate care at a community hospital as an alternative to prolonged general hospital care for elderly patients: a randomised controlled trial. BMC Public Health. 2007; 7(1):68

3. Glasby J, Martin G, Regen E. Older people and the relationship between hospital services and intermediate care: Results from a national evaluation. J Interprof Care. 2008; 22(6):639-649.

4. Mendes EV. As redes de atenção à saúde. Ciênc. Saúde Colet. 2010; 15(5):2297-2305.

5. World Health Organization, editor. Innovative care for chronic conditions: building blocks for action: global report. Geneva: World Health Organization; 2003.

6. Grant JA, Dowell J. A qualitative study of why general practitioners admit to community hospitals. $\mathrm{Br} \mathrm{J}$ Gen Pract. 2002; 52(481):628-30, 632-5.

7. Garåsen H, Windspoll R, Johnsen R. Long-term patients' outcomes after intermediate care at a community hospital for elderly patients: 12-month follow-up of a randomized controlled trial. Scand J Public Health. 2008; 36(2):197-204.

8. Green J, Young J, Forster A, et al. Effects of locality based community hospital care on independence in older people needing rehabilitation: randomised controlled trial. BMJ. 2005; 331(7512):317-322.

9. Brasil. Ministério da Saúde. Portaria no 2.809, de 7 de dezembro de 2012. Estabelece a organização dos Cuidados Prolongados para retaguarda à Rede de Atenção às Urgências e Emergências (RUE) e às demais Redes Temáticas de Atenção à Saúde no âmbito do Sistema Único de Saúde (SUS). Diário Oficial da União. 10 Dez 2012.
10. Souza M, Silva MD, Carvalho R. Integrative review: what is it? How to do it? Einstein (Sao Paulo). 2010; 8(1):102-106.

11. Suber P. Opening Access to Research. Open Society Foundations [internet]. 2012 [acesso em 2019 jul 8]. Disponível em: https://www.opensocietyfoundations. org/voices/opening-access-research.

12. Dixon S, Kaambwa B, Nancarrow S, et al. The relationship between staff skill mix, costs and outcomes in intermediate care services. BMC Health Serv Res. 2010; 10(1).

13. Higgs R. Example of intermediate care: the new Lambeth Community Care Centre. BMJ. 1985; 291(6506):1395-1397.

14. Kilgore C. Why intermediate care services need to be refreshed: A national audit of this form of provision has revealed a wide variation in capacity, staffing and resources. Louise Hunt discusses where the proble$\mathrm{ms}$ lie and how they could be overcome. Nurs Older People. 2014; 26(3):16-20.

15. Vetter NJ. Commentary: Intermediate care: policy before evidence. BMJ. 2005; (331):1.

16. Millar A, Hughes C, Devlin M, et al. A cross-sectional evaluation of community pharmacists' perceptions of intermediate care and medicines management across the healthcare interface. Int J Clin Pharm. 2016; 38(6):1380-1389.

17. Gladman J, Buckell J, Young J, et al. Understanding the Models of Community Hospital rehabilitation Activity (MoCHA): a mixed-methods study. BMJ Open. 2017; 7(2):e010483.

18. Kaambwa B, Bryan S, Barton P, et al. Costs and health outcomes of intermediate care: results from five UK case study sites: Cost and outcomes of intermediate care. Health Soc Care Community. 2008; 16(6):573581. 
19. Oliver D. David Oliver: Improving access to intermediate care. BMJ. 2017; (356):i6763

20. Regen E, Martin G, Glasby J, et al. Challenges, benefits and weaknesses of intermediate care: results from five UK case study sites. Health Soc Care Community. 2008; 6(6):629-637.

21. Seamark D, Moore B, Tucker H, et al. Community hospitals for the new millennium. Br J Gen Pract. 2001; (51):125-127.

22. Williams KS, Kurtev S, Phelps K, et al. Shifting care from community hospitals to intensive community support: a mixed method study. Prim Health Care Res Dev. 2018; 19(01):53-63.

23. Yohannes AM, Baldwin RC, Connolly MJ. Prevalence of depression and anxiety symptoms in elderly patients admitted in post-acute intermediate care. Int J Geriatr Psychiatry. 2008; 23(11):1141-1147.

24. Young J, Gladman JRF, Forsyth DR, et al. The second national audit of intermediate care. Age Ageing. 2015; 44(2):182-184.

25. Dahl U, Johnsen R, Sætre R, et al. The influence of an intermediate care hospital on health care utilization among elderly patients - a retrospective comparative cohort study. BMC Health Serv Res. 2015; 15(1).

26. Dahl U, Steinsbekk A, Johnsen R. Effectiveness of an intermediate care hospital on readmissions, mortality, activities of daily living and use of health care services among hospitalized adults aged 60 years and older-a controlled observational study. BMC Health Serv Res. 2015; 15(1).

27. Garåsen H, Magnussen J, Windspoll R, et al. [Elderly patients in hospital or in an intermediate nursing home department - cost analysis]. Tidsskr Nor Legeforen. 2008; 128(3):283-285.

28. Lappegard Ø, Hjortdahl P. Perceived quality of an alternative to acute hospitalization: An analytical study at a community hospital in Hallingdal, Norway. Soc Sci Med. 2014; (119):27-35.
29. Colprim D, Martin R, Parer M, et al. Direct Admission to Intermediate Care for Older Adults With Reactivated Chronic Diseases as an Alternative to Conventional Hospitalization. J Am Med Dir Assoc. 2013; 14(4):300-302.

30. Inzitari M, Espinosa Serralta L, Pérez Bocanegra MC, et al. Derivación de pacientes geriátricos subagudos a un hospital de atención intermedia como alternativa a la permanencia en un hospital general. Gac Sanit. 2012; 26(2):166-169.

31. Santaeugènia SJ, Tomás S, Álvaro M, et al. Direct Admission to Intermediate Care for Older Adults With Reactivated Chronic Diseases: Avoiding Both Conventional Hospitalization and Emergency Department Use? J Am Med Dir Assoc. 2013; 14(6):444-445.

32. Santaeugènia SJ, García-Lázaro M, Alventosa AM, et al. Nuevo modelo de atención integrada orientada a ancianos ingresados en Unidades de Atención Intermedia en Cataluña: protocolo de un estudio cuasiexperimental. Rev Esp Geriatr Gerontol. 2017; 52(4):201-208.

33. Martinsen B, Norlyk A, Lomborg K. Experiences of intermediate care among older people: a phenomenological study. Br J Community Nurs. 2015; 20(2):74-79.

34. Milte CM, Ratcliffe J, Davies O, et al. Family meetings for older adults in intermediate care settings: the impact of patient cognitive impairment and other characteristics on shared decision making. Health Expect. 2015; 18(5):1030-1040.

35. Pianori D, Avaldi VM, Rosa S, et al. How do Community Hospitals respond to the healthcare needs of elderly patients? A population-based observational study in the Emilia-Romagna Region. Ann Ig. 2018; (4):317-329.

36. Itália. Decreto $\mathrm{n}^{\circ} 70,2$ aprile 2015. Regolamento recante definizione degli standard qualitativi, strutturali, tecnologici e quantitativi relativi all'assistenza ospedaliera. Gazzetta Ufficiale. 2015 abr 2. [acesso em 2019 ago 28]. Disponível em: https://www.gazzettaufficiale.it/eli/id/2015/06/04/15G00084/sg. 
37. Franklin DJ, Cheville AL. Medical Rehabilitation and the Palliative Care Patient. In: Oxford Textbook of Palliative Medicine. 5. ed. Vol 1. Oxford: Oxford University Press; 2015. p. 1-31.

38. Tiberini R, Richardson H. Rehabilitative Palliative Care: enabling people to live fully until they die: a Challenge for the 21st Century. London: Hospice UK; 2015 [acesso em 2021 maio 24]. Disponível em: https://www.hospiceuk.org/what-we-offer/clinical-and-care-support/rehabilitative-palliative-care/resources-for-rehabilitative-palliative-care.
39. Souza Filho BAB, Tritany ÉF. Cuidados Paliativos: uma abordagem transversal para a Rede de Atenção à Saúde. In: Franco TB, Mendonça PEX, Conceição MR, et al., organizadores. Cuidados Intermediários e Redes de Atenção à Saúde. Porto Alegre: Rede Unida; 2020. p. 236-251.

Recebido em 14/06/2020

Aprovado em 05/11/2020

Conflito de interesses: inexistente

Suporte financeiro: Coordenação de Aperfeiçoamento de Pessoal de Nível Superior (Capes) 